\section{BMJ Open Respiratory Research}

\title{
Analyses of abdominal adiposity and metabolic syndrome as risk factors for respiratory distress in COVID-19
}

Cathelijne M van Zelst (D) , ${ }^{1,2}$ Matthijs L Janssen, ${ }^{2,3}$ Nadine Pouw, ${ }^{4}$ Erwin Birnie,${ }^{5,6}$ Manuel Castro Cabezas, ${ }^{7}$ Gert-Jan Braunstahl ${ }^{1,2}$

\section{ABSTRACT}

To cite: van Zelst CM, Janssen ML, Pouw N, et al. Analyses of abdominal adiposity and metabolic syndrome as risk factors for respiratory distress in COVID-19. BMJ Open Resp Res 2020;7:e000792. doi:10.1136/ bmjresp-2020-000792

- Additional material is published online only. To view please visit the journal online (http://dx.doi.org/10.1136/ bmjresp-2020-000792).

CMvZ and MLJ contributed equally.

Received 2 October 2020 Revised 18 November 2020 Accepted 20 November 2020
Background Several characteristics of the metabolic syndrome, such as obesity and hypertension, have emerged as risk factors for a poor clinical outcome in COVID-19. However, most reports lack data on the metabolic syndrome itself. This study investigated prospectively the relationship between respiratory deterioration and the presence of metabolic syndrome or abdominal adiposity in patients with COVID-19. Methods A prospective observational cohort study analysing patients with respiratory symptoms who presented at a local emergency department in the Netherlands. The influence of abdominal adiposityassessed by an increased waist-hip ratio-and metabolic syndrome on respiratory deterioration and the length of hospital stay were analysed with multivariable logistic regressions and Kaplan-Meier analyses.

Results In total, 166 patients were analysed, of whom $86(52 \%)$ tested positive for COVID-19. The prevalence of metabolic syndrome did not differ between patients with COVID-19 with and without the need for intubation or level of supportive care ( $37.5 \%$ vs $48.4 \%, p=0.338$ ). In contrast, abdominal adiposity is an independent risk factor for respiratory distress in COVID-19, adjusted for metabolic syndrome, age, gender and $\mathrm{BMI}(\mathrm{OR} 1.11,95 \% \mathrm{Cl} 1.02$ to $1.20, p=0.014$ ).

Conclusion This study shows that abdominal adiposity, and not the presence of metabolic syndrome, is associated with clinical deterioration in COVID-19. This prospective study provides further insight into the risk stratification of patients with COVID-19 based on a simple measurement as the waist and hip circumference. Trial registration number NL8580.

\section{INTRODUCTION}

Since the outbreak of COVID-19 (SARS-CoV-2) in China in the winter of 2019, the disease has spread rapidly, causing a pandemic. Although the vast majority of patients has only mild upper airway symptoms, a significant proportion of patients suffers from clinically relevant respiratory distress and requires hospitalisation. Several retrospective cohort studies have described the characteristics of admitted patients, thereby providing insight in the subgroups at

\section{Key question \\ Is the metabolic syndrome and/or abdominal adiposity associated with respiratory distress in COVID-19? \\ Bottom line \\ - In contrast to the metabolic syndrome, it was shown that abdominal adiposity, as measured by an in- creased waist-hip ratio, is strongly associated with an unfavourable outcome in COVID-19. \\ Why read on \\ - In an extensive COVID-19 study, the presence of met- abolic syndrome and abdominal adiposity measured non-invasively by waist and hip circumference, lev- els of adipocytokine and duration of hospitalisation were analysed and related to oxygen demand.}

risk for clinical deterioration. It was observed that, among others, obesity, diabetes and hypertension were prevalent comorbidities in patients hospitalised with COVID-19. ${ }^{1-3}$ The majority of patients suffering from COVID-19 who were admitted to the intensive care unit (ICU) or deceased had at least one of these comorbidities. ${ }^{45}$ Moreover, obesity is a significant independent risk factor for respiratory failure in COVID-19, ${ }^{6}$ as observations in 2009 influenza A infection reveal. ${ }^{7}$ An explanation for this observation was suggested to be a prolonged viral excretion in patients with obesity. ${ }^{8}$ Surprisingly, despite a high prevalence of obesity among patients with hypertension and diabetes, ${ }^{9}$ most reports lack data on the outcome of COVID-19 in patients with and without the metabolic syndrome (MetS). Recently published research suggests that intra-abdominal fat depositions (representing abdominal adiposity) on imaging studies are associated with an unfavourable outcome in COVID-19. ${ }^{10} 11$

Visceral fat depositions secrete more inflammatory cytokines, causing an imbalance in the anti-inflammatory and proinflammatory adipokines, thereby altering the immune 
response. An imbalance in adipokines, resulting in an elevated leptin-adiponectin ratio, is related to increasing insulin resistance. ${ }^{12}$ Overexpression of proinflammatory cytokines, such as leptin and interleukin 6 (IL-6), causes oxidative stress and endothelial dysfunction. ${ }^{13}$ Recent literature showed that the levels of IL-6, which is also partly derived from adipose tissue, are elevated in patients with COVID-19 with obesity and severe respiratory distress admitted to the ICU. ${ }^{14}{ }^{15}$ Possibly, this might play a role in the described 'cytokine storm' in patients with COVID-19, thereby making patients with obesity susceptible to respiratory failure.

It has been recognised that the association between the type of obesity, insulin resistance and COVID-19 should be investigated in detail. ${ }^{1617}$ In the current study, we investigated the association between MetS, separate criteria of the MetS and severity of disease course in COVID-19 in terms of the required level of respiratory support and the length of hospital stay. We hypothesised that patients with MetS are at risk for an unfavourable course of COVID-19 within 30 days after presentation at the emergency room (ER).

\section{METHODS}

\section{Study design and participants}

The MASC study (acronym of Metabolic syndrome And Severity of COVID-19) is a prospective cohort study, conducted from 16 April 2020 until 23 May 2020 at the Franciscus Gasthuis \& Vlietland, Rotterdam, the Netherlands. Consecutive patients, aged $\geq 18$ years, presenting with respiratory symptoms or fever suspect for having COVID-19, were assessed for inclusion. The presence of COVID-19 was confirmed by means of PCR performed on patient material obtained by a nasopharyngeal swab or serologic tests. Patients presenting with respiratory symptoms who repeatedly tested negative on COVID-19 PCR but positive for SARS-CoV-2 antibodies were considered positive for COVID-19. Patients with a 'do not resuscitate/intubate' order, patients unable to stand upright (due to respiratory distress or pre-existent comorbidities) or patients without measurements of hip and waist circumference were excluded. Patients were followed prospectively for 30 days after presentation. By order of the local research board, only oral consent but no written patient consent was required for performing measurements.

\section{Outcomes}

\section{Primary outcome}

Unfavourable outcome of disease was defined as being admitted to the hospital with requirement of maximum respiratory support of $3 \mathrm{~L} /$ minute $(\mathrm{min})$ supplemental oxygen or more at any time during follow-up, requirement of supplemental oxygen by means of high-flow nasal cannula intubation or admission to the ICU.

Favourable outcome of disease was defined as being discharged from the hospital having required less than
$3 \mathrm{~L} / \mathrm{min}$ oxygen or no admission to the hospital ward at all. Demand for supplemental oxygen was defined as having an oxyhaemoglobin saturation below $94 \%$ on room air.

\section{Secondary outcomes}

Length of stay in hospital: measured in days with a follow-up time of 30 days after referral to the ER.

\section{Definitions}

The presence of MetS was defined as the presence of any three of the following five traits, modified after the Adult Treatment Panel III criteria ${ }^{18}$ :

- Abdominal adiposity, defined as a waist circumference $\geq 102 \mathrm{~cm}$ in men and $\geq 88 \mathrm{~cm}$ in women, measured in the upright position.

- Serum triglycerides $\geq 1.7 \mathrm{mmol} / \mathrm{L}$ or treatment with lipid-lowering drugs.

- Serum high-density lipoprotein cholesterol (HDL-C) $<1 \mathrm{mmol} / \mathrm{L}$ in men and $<1.3 \mathrm{mmol} / \mathrm{L}$ in women.

- Drug treatment for elevated blood pressure.

- Non-fasting plasma glucose $(>7.8 \mathrm{mmol} / \mathrm{L})$ or drug treatment for elevated blood glucose.

Anthropometric characteristics: abdominal adiposity was assessed by circumferences measurements of waisthip ratio as defined according to WHO. ${ }^{19}$

In order to provide a biochemical explanation for the influence of abdominal adiposity, IL-6 and adipocytokine (adiponectin and leptin) levels were measured in blood samples that were drawn at ER admission.

\section{Data collection}

On presentation, baseline characteristics, clinical, laboratory and radiological data were collected prospectively following a predefined study protocol. Key data on demographics, baseline comorbidities and presenting clinical parameters were obtained for all included patients. During physical examination at ER presentation, measurements of waist and hip circumference, height and weight were obtained and blood was drawn for routine medical care and cytokine analyses. Detailed data on clinical outcomes, such as supportive care, (duration of) admission, ICU admission and survival were registered at the end of the 30-day follow-up period. Baseline and outcome data were obtained by means of standardised data collection forms.

Laboratory measurements were carried out at the Department of Clinical Chemistry, Franciscus Gasthuis \& Vlietland (Rotterdam, the Netherlands) according to standard procedures. IL-6, adiponectin and leptin heparin plasma concentrations were determined using a commercially available ELISA kit (R\&D Systems, Minneapolis, Minnesota, USA), according to the manufacturer's instructions. The minimum detectable dose (MDD) of the ELISA assays was $0.70 \mathrm{pg} / \mathrm{mL}$ (IL-6), $0.891 \mathrm{ng}$ / $\mathrm{mL}$ (adiponectin) and $7.8 \mathrm{pg} / \mathrm{mL}$ (leptin), respectively. 
Samples with a concentration lower than the MDD were excluded from analyses.

\section{Patient and public involvement section}

Patients were not involved in study design, but the purpose of the study measurements was explained just before measurement. As (abdominal) adiposity and MetS (criteria) are both relevant public health issues, no patients or public involvement was applicable in development of the research questions. Patients were politely invited for participation and offered the option to decline participation without further explanation or the reason why. Patients or public were not involved in design/conduct of the study, choice of outcome measures or recruitment of other patients. Patients/public were not involved in publication/dissemination of the study results.

\section{Statistical analyses}

Baseline data were compared between prespecified subgroups based on the presence of COVID-19, MetS and favourable or unfavourable outcome. Patients in whom data were insufficient to assess the presence of MetS (see definition abovementioned) were excluded from comparisons between patients with and without MetS. Continuous variables were expressed as median with IQR and differences between groups compared with the Mann-Whitney U test (non-normally distributed variables) or unpaired t-test (normally distributed variables); categorical variables were expressed as number with percentages $(\%)$ and compared between groups by $\chi^{2}$ test. A two-sided $\mathrm{p}$ of $<0.05$ was considered a statistically significant difference.

Univariable and multivariable binary logistic regression analysis were used to study the association between covariates and the course of disease. This was expressed by ORs,

Total number of patients supected for COVID-19 at ER in inclusion period: $\mathbf{n}=\mathbf{5 0 7}$

\begin{tabular}{|l|}
\hline $\begin{array}{l}\text { Excluded: } \mathbf{n}=\mathbf{1 0 4} \\
\text { Patients unable to stand upright } \\
\text { because of respiratory distress: } \mathbf{n}=\mathbf{2 0} \\
\text { Patients with a "do not resuscitate/ } \\
\text { intubate" order: } \mathbf{n}=\mathbf{8 4}\end{array}$ \\
$\begin{array}{l}\text { Excluded: } \mathbf{n}=\mathbf{2 3 6} \\
\text { Unable to stand upright because of } \\
\text { comorbidities; } \\
\text { Lack of data, e.g. no hip and waist } \\
\text { circumference measurement }\end{array}$ \\
\hline
\end{tabular}

Included patients: $\mathbf{n = 1 6 6}$

Lost to follow-up: $\mathbf{n}=\mathbf{0}$

Analyzed: $\mathbf{n = 1 6 6}$

Figure 1 Patient enrolment. ER, emergency room.
95\% CI and $p$ values. Goodness of model fit was based on the model $\chi^{2}$ ( $p$ value). In additional analyses, each of the five criteria of the MetS, waist-hip ratio and BMI is adjusted for age and gender in multivariable logistic regression models. Variables with $\mathrm{p}<0.10$ in univariable regression were considered as relevant covariates to be included in a final multivariable postadjusted regression model. Based on an a priori scientific understanding that male gender and increasing age are risk factors for an unfavourable disease course in COVID-19, these variables were included in the multivariable regression regardless of the statistical significance in univariable regression. To avoid overestimation, not more than five variables were included in the final multivariable logistic regression. Multicollinearity (correlation between waist-hip ratio and gender) was checked. Potential confounding was evaluated using stratified analysis. A receiver operating characteristic (ROC) curve was used to validate the discriminative ability of the multivariable logistic regression model. A sample size calculation was up front not possible, therefore a post hoc statistical power for the multiple regression was calculated.

The association between MetS and length of stay in the hospital was analysed by means of univariable and multivariable Cox regression. An event was defined as being discharged from hospital alive, within the follow-up period (30 days after hospital admission). Patients who were still admitted and the end of follow-up were censored at day 30. Again, multivariable regression was performed to adjust for possible imbalances between groups. Based on a priori scientific understanding, the same variables as in multivariable logistic regression were included. Covariates that were statistically significant and/or closely associated in univariable analysis were included in the multivariable model. Kaplan-Meier curves were constructed and stratified by presence of MetS or increased waist-hip ratio (according to WHO criteria $^{19}$ ) and unfavourable outcome. Data were analysed using IBM SPSS Statistics V.26.

\section{RESULTS}

\section{Descriptive data}

From 506 patients presenting at the ER with suspicion of COVID-19, 166 patients were included in the analysis. Patients unable to stand upright because of respiratory distress $(n=20)$, patients with a 'do not resuscitate/ intubate' order $(n=84)$, patients with a lack of data (no hip and waist circumference measurement) or patients unable to stand upright because of comorbidities $(\mathrm{n}=236)$ were excluded for analyses (figure 1$)$. Of the 166 included patients, $86(52 \%)$ tested positive for COVID-19, of whom COVID-19 diagnosis was based on a positive PCR in $80(93 \%)$ and a positive antibody test in six patients $(7 \%)$. Due to missing data, presence of MetS could not be determined in seven patients, who were therefore excluded from comparisons between favourable and unfavourable groups. 


\begin{tabular}{|c|c|c|c|}
\hline & COVID-19 negative $(n=79)$ & COVID-19 positive $(n=86)$ & $P$ value \\
\hline Male, N (\%) & $33(41.8)$ & $40(46)$ & 0.586 \\
\hline Age, years, median (IQR) & $60(45-67)$ & $56(47-61.5)$ & 0.267 \\
\hline $\mathrm{BMI}$ in $\mathrm{kg} / \mathrm{m}^{2}$, median (IQR) & $26.1(23.1-29.3)$ & $27.8(24.7-32.9)$ & 0.005 \\
\hline Metabolic syndrome, N (\%) & $26(41.9)$ & $33(38.7)$ & 0.984 \\
\hline Use of antihypertensives, $\mathrm{N}(\%)^{*}$ & $25(36.2)$ & $22(25.6)$ & 0.152 \\
\hline High triglycerides, $\mathrm{N}(\%)^{\star}$ & $37(48.7)$ & $29(34.5)$ & 0.069 \\
\hline Low HDL-C, N (\%)* & $28(36.8)$ & $69(84.1)$ & $<0.001$ \\
\hline Hyperglycaemia, N (\%)* & $19(24.4)$ & $25(28.7)$ & 0.526 \\
\hline Abdominal adiposity, $\mathrm{N}(\%)^{\star}$ & $46(60.5)$ & $59(68.6)$ & 0.283 \\
\hline Waist-hip ratio, median (IQR) & $0.96(0.87-1.02)$ & $0.94(0.88-1.00)$ & 0.492 \\
\hline History of pulmonary disease, $\mathrm{N}(\%)$ & $35(44.3)$ & $18(20.7)$ & 0.001 \\
\hline History of cardiovascular disease, N (\%) & $18(22.8)$ & $18(20.7)$ & 0.744 \\
\hline
\end{tabular}

Median (IQR Q1-Q3) used in variables with non-normal distribution. Statistically significant p-values are bold.

*Metabolic syndrome criterion. Cut-off values: high triglycerides $(>1.7 \mathrm{mmol} / \mathrm{L})$, low $\mathrm{HDL}-\mathrm{C}(<1 \mathrm{mmol} / \mathrm{L}$ in $\mathrm{male},<1.3 \mathrm{mmol} / \mathrm{L}$ in female), hyperglycaemia $\geq 7.8 \mathrm{mmol} / \mathrm{L}$ and/or drug treatment for elevated blood glucose, abdominal adiposity (male $\geq 102 \mathrm{~cm}$, female $\geq 88 \mathrm{~cm}$ ).

BMI, body mass index; HDL-C, high-density lipoprotein cholesterol; N, numbers.

The proportion of patients with MetS was equal in patients positive for COVID-19 and negative for COVID-19 (table $1,38.7 \%$ vs $41.9 \%, \mathrm{p}=0.984$ ). The COVID-19-positive group had a significantly higher median BMI (27.8 vs $\left.26.1 \mathrm{~kg} / \mathrm{m}^{2}, \mathrm{p}=0.005\right)$ and a higher proportion of patients with low HDL-C $(84.1 \%$ vs $36.8 \%$, $\mathrm{p}<0.001)$ compared with the negative group.

Table 2 shows the clinical parameters of patients with $(n=33)$ and without $(n=46)$ MetS. Number of MetS criteria divided in favourable and unfavourable outcome are shown in online supplemental figure S1. A history of cardiovascular disease was more prevalent among patients with MetS compared with those without MetS (33.3\% vs $10.9 \%, \mathrm{p}=0.014)$. All patients with COVID-19 and MetS and $89 \%$ of patients with COVID-19 without MetS were hospitalised $(\mathrm{p}=0.047)$. The prevalence of MetS did not differ significantly between patients with a favourable and unfavourable outcome $(37.5 \%$ vs $48.4 \%$, $\mathrm{p}=0.338$ ). Among patients with an unfavourable outcome of COVID-19 infection, abdominal adiposity was significantly more prevalent compared with those with a favourable outcome $(82.9 \%$ vs $58.0 \%, \mathrm{p}=0.015)$.

\section{Predictive model for unfavourable outcome}

Table 3 shows the results from univariable and multivariable postadjusted logistic regression, analysing the odds of an unfavourable outcome in COVID-19. MetS and all the separate criteria of MetS were also analysed in additional logistic regressions, adjusted for age and gender, showing significant impact on the outcome for both abdominal adiposity and also for BMI (online supplemental table S1). The final multivariable model shows that the waist-hip ratio (OR 1.11, 95\% CI 1.02 to $1.20, \mathrm{p}=0.014)$ and BMI (OR $1.11,95 \%$ CI 1.00 to $1.23, \mathrm{p}=0.043$ ) were significantly associated with an increased risk for an unfavourable outcome of
COVID-19, when adjusted for MetS, age and gender. MetS was not significantly related to an unfavourable outcome in univariable and multivariable logistic regressions. Online supplemental figure 2 shows that the area under the ROC curve was 0.771 (95\% CI: 0.664 to 0.878 ) and post hoc statistical power analysis for this model is 0.85 .

\section{Length of hospital stay}

No patients died during the 30-day follow-up period (table 2). Figure 2 shows duration of hospitalisation for patients positive for COVID-19, with and without MetS. The median (IQR) time until discharge was 6 (3-8) days in the MetS group and 5 (3-11) days in the group without MetS (log-rank test $\mathrm{p}=0.921)$. Figure 3 represents the Kaplan-Meier curves of patients with COVID-19 with and without abdominal adiposity based on waist-hip ratio. Median time until discharge was 4 days in the group without abdominal adiposity (IQR 2-8) and 6 days in the group with abdominal adiposity (IQR 3-11). There was no statistically significant difference between the curves ( $\log$-rank test $\mathrm{p}=0.129)$.

Table 4 shows the univariable and multivariable association between patient characteristics and length of stay using Cox regression. In univariable Cox regression, MetS was not significantly related to length of hospital stay $(\mathrm{HR}=0.98,95 \%$ CI 0.80 to $2.22, \mathrm{p}=0.23)$. Waist-hip ratio indicated a prolonged length of hospital stay in the univariable Cox regression model ( $\mathrm{HR}=0.98,95 \%$ CI 0.95 to $0.99, \mathrm{p}=0.04$ ). However, it did not show a statistically significant effect on length of stay, when adjusted for age and gender $(\mathrm{p}=0.29)$.

\section{Cytokine measurement}

In a subset of patients, the levels of proinflammatory (IL-6, leptin) and anti-inflammatory cytokines (adiponectin) 


\begin{tabular}{|c|c|c|c|}
\hline & Without MetS $\mathrm{n}=46$ & MetS $\mathrm{n}=\mathbf{3 3}$ & $P$ value \\
\hline Male, N (\%) & $23(50.0)$ & $14(42.4)$ & 0.506 \\
\hline Age, years, median (IQR) & $48.5(34-60.3)$ & $56.0(45.3-61.8)$ & 0.107 \\
\hline $\mathrm{BMI}$ in $\mathrm{kg} / \mathrm{m}^{2}$, median (IQR) & $26.5(23.8-31.4)$ & $30.4(27.2-35.8)$ & 0.004 \\
\hline MetS, N (\%) & - & - & - \\
\hline Use of antihypertensives, $\mathrm{N}(\%)^{*}$ & $4(8.7)$ & $17(51.5)$ & - \\
\hline High triglycerides, $\mathrm{N}(\%)^{\star}$ & $2(4.3)$ & $24(72.7)$ & - \\
\hline Low HDL-C, N (\%)* & $36(87.3)$ & $33(100)$ & - \\
\hline Hyperglycaemia, N (\%)* & $2(4.3)$ & $19(57.6)$ & - \\
\hline Abdominal adiposity, $\mathrm{N}(\%)^{*}$ & $24(52.2)$ & $29(87.9)$ & - \\
\hline Waist-hip ratio, median (IQR) & $0.93(0.84-0.98)$ & $0.95(0.90-1.02)$ & 0.034 \\
\hline History of pulmonary disease, $\mathrm{N}(\%)$ & $10(21.7)$ & $6(18.2)$ & 0.698 \\
\hline History of cardiovascular disease, N (\%) & $5(10.9)$ & $11(33.3)$ & 0.014 \\
\hline Use of antibiotics, $N(\%)$ & $19(41.3)$ & $10(30.3)$ & 0.274 \\
\hline Deceased (30-day follow-up), N (\%) & $0(0)$ & $0(0)$ & - \\
\hline Discharged from ER without hospitalisation, N (\%) & $5(11)$ & $0(0)$ & 0.047 \\
\hline Hospitalisation, N (\%) & $41(89)$ & $33(100)$ & \\
\hline \multicolumn{4}{|l|}{ Respiratory support† } \\
\hline Intubation, N (\%) & $7(15)$ & $6(18)$ & \multirow[t]{3}{*}{0.989} \\
\hline High-flow nasal cannula, N (\%) & $4(9)$ & $3(9)$ & \\
\hline Supplemental oxygen 1-6 L/min, N (\%) & $24(52)$ & $21(64)$ & \\
\hline 6 & - & - & \multirow[t]{7}{*}{0.111} \\
\hline 5 & $1(2)$ & $2(6)$ & \\
\hline 4 & 0 & $3(9)$ & \\
\hline 3 & $4(9)$ & $1(3)$ & \\
\hline 2 & $9(20)$ & $11(33)$ & \\
\hline 1 & $10(22)$ & $3(9)$ & \\
\hline Hospitalised, without oxygen supply, N (\%) & $6(13)$ & $4(12)$ & \\
\hline Unfavourable course of disease, $\mathrm{N}(\%)$ & $16(35)$ & $15(45)$ & 0.338 \\
\hline Readmission ER related to COVID-19, N (\%) & $7(15)$ & $4(12)$ & 0.658 \\
\hline
\end{tabular}

Sufficient data to diagnose or exclude MetS were missing in seven patients. Median (IQR Q1-Q3) used in variables with non-normal distribution. Statistically significant $p$-values are bold.

*MetS criterion. Cut-off values: high triglycerides ( $>1.7 \mathrm{mmol} / \mathrm{L})$, low HDL-C ( $<1 \mathrm{mmol} / \mathrm{L}$ in male, $<1.3 \mathrm{mmol} / \mathrm{L}$ in female), hyperglycaemia $\geq 7.8 \mathrm{mmol} / \mathrm{L}$ and/or drug treatment for elevated blood glucose, abdominal adiposity (male $\geq 102 \mathrm{~cm}$, female $\geq 88 \mathrm{~cm}$ ).

†Respiratory support was defined as maximum supplemental oxygen at any given moment during hospitalisation.

BMI, body mass index; ER, emergency room; HDL-C, high-density lipoprotein cholesterol; MetS, metabolic syndrome; N, numbers.

were measured at ER admission and compared between groups (table 5, figure 4). Plasma samples for cytokine measurement were available from 29 included patients (12 COVID-19-positive and 17 COVID-19-negative). Baseline characteristics were comparable between these two groups table 5Comparison between patients with COVID-19 with and without MetS showed that IL-6 was lower in patients with COVID-19 with MetS (median 70.01 vs $30.60 \mathrm{pg} / \mathrm{mL}, \mathrm{p}=0.028$ ). IL-6 was higher in patients with COVID-19 with an unfavourable outcome than in those without (median 94.7 vs 30.7, $\mathrm{p}=0.034$ ). The leptin-adiponectin ratio was higher among patients with COVID-19 with MetS compared with those without MetS (ratio 6.6 vs $1.9, \mathrm{p}=0.003$, table 5 . This was not observed when comparing the ratio between patients with an unfavourable outcome to patients with a favourable outcome $(\mathrm{p}=0.943)$.

\section{DISCUSSION}

This study is the first to investigate the influence of abdominal adiposity assessed by the waist-hip circumference, a non-invasive measurement, on COVID-19 outcome. Abdominal adiposity was significantly associated with a more severe course of COVID-19, adjusted for age, gender and BMI. Similar observations are made in recently published studies, analysing the amount of intra-abdominal (eg. visceral) fat depositions in patients with COVID-19. ${ }^{11}$ Agreeing with the present study, these investigations show that abdominal adiposity on 
Table 3 Univariable and multivariable postadjusted logistic regression analyses: association between patient characteristics and severity of COVID-19

\begin{tabular}{|c|c|c|c|c|}
\hline \multirow[b]{2}{*}{ Covariate } & \multicolumn{2}{|c|}{ Univariable logistic regression } & \multicolumn{2}{|c|}{$\begin{array}{l}\text { Final multivariable postadjusted } \\
\text { logistic regression model }\end{array}$} \\
\hline & OR $(95 \% \mathrm{Cl})$ & $P$ value & OR $(95 \% \mathrm{Cl})$ & $P$ value \\
\hline Constant & - & - & 0.000 & 0.001 \\
\hline Male gender & 2.28 (0.95 to 5.48$)$ & 0.064 & 0.90 (0.23 to 3.56$)$ & 0.884 \\
\hline Age & 1.02 (0.98 to 1.06$)$ & 0.288 & 1.01 (0.96 to 1.06$)$ & 0.684 \\
\hline Metabolic syndrome & $1.56(0.63$ to 3.90$)$ & 0.339 & $0.70(0.21$ to 2.19$)$ & 0.506 \\
\hline Use of antihypertensives* & $0.58(0.21$ to 1.63$)$ & 0.303 & - & - \\
\hline Hypertriglyceridemia* & $1.13(0.45$ to 2.83$)$ & 0.802 & - & - \\
\hline Low HDL-C* & 2.63 (0.67 to 10.42$)$ & 0.168 & - & - \\
\hline Hyperglycaemia* & 1.79 (0.70 to 4.58$)$ & 0.225 & - & - \\
\hline Abdominal adiposity* & 3.50 (1.23 to 9.93$)$ & 0.019 & - & - \\
\hline Waist-hip ratio & 1.11 (1.05 to 1.18$)$ & 0.001 & $1.11(1.02$ to 1.20$)$ & 0.014 \\
\hline BMI & $1.11(1.02$ to 1.21$)$ & 0.016 & $1.11(1.00$ to 1.23$)$ & 0.043 \\
\hline Post hoc power & - & & 0.85 & \\
\hline
\end{tabular}

*Metabolic syndrome criterion. Cut-off values: high triglycerides ( $>1.7 \mathrm{mmol} / \mathrm{L})$, low HDL-C ( $<1 \mathrm{mmol} / \mathrm{L}$ in male, $<1.3 \mathrm{mmol} / \mathrm{L}$ in female), hyperglycaemia $\geq 7.8 \mathrm{mmol} / \mathrm{L}$ and/or drug treatment for elevated blood glucose, abdominal adiposity (male $\geq 102 \mathrm{~cm}$, female $\geq 88 \mathrm{~cm}$ ). Statistically significant $p$-values are bold.

$\mathrm{BMI}$, body mass index; HDL-C, high-density lipoprotein cholesterol.

CT imaging is related to respiratory failure in COVID19. However, our results are based on measurement of the waist-hip circumference, which is compared with abdominal CT scanning, a clinically more feasible and non-invasive method to assess abdominal adiposity. Overweight $(>50 \%)$ and obesity $(15.9 \%)$ are highly prevalent in the general Dutch population. ${ }^{20}$ Patients presenting with respiratory symptoms at our centre who tested positive for COVID-19 had a higher BMI compared with those without COVID-19. Given that (abdominal) adiposity is a treatable risk factor, in contrast to other risk factors such as age and gender, our results underline the relevance of this topic in COVID-19 research. In accordance with

\section{Survival Analysis: time until discharge}

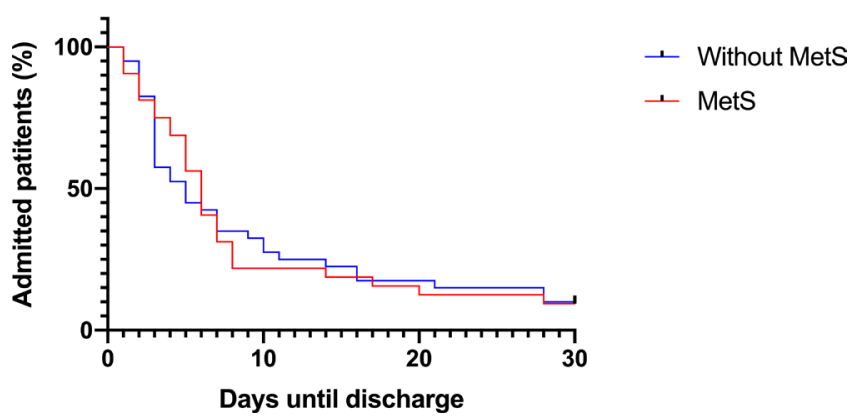

Figure 2 Survival analysis displaying time until discharge in patients with COVID-19 compared between patients with and without metabolic syndrome (MetS). Median time until discharge was 6 days in the MetS group (IQR 3-8) and 5 days in the group without MetS [(IQR 3-11). Log-rank test $p$ value $=0.921$. Patients who were still admitted and the end of the follow-up period were censored at day 30 . No patients died during follow-up. recent literature, the present study also describes a relationship between BMI and respiratory failure in COVID19. But, according to our study, abdominal adiposity seems to be a more important risk factor for an unfavourable outcome in COVID-19. This is in line with earlier reports suggesting that abdominal adiposity is a strong predictor for respiratory deterioration in COVID-19 and in conditions other than COVID-19. ${ }^{101121-23}$

The association between MetS and the severity of COVID-19 infection was investigated in this study, which had never been done before. It was observed that all patients with COVID-19 who fulfilled the criteria for MetS

Survival Analysis: time until discharge

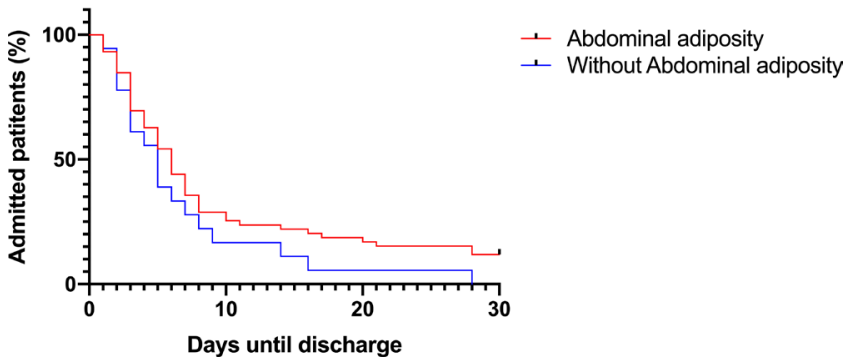

Figure 3 Survival analysis displaying time until discharge in patients with COVID-19, compared between patients with and without abdominal adiposity. Abdominal adiposity is defined according to $\mathrm{WHO}$ criteria male $>0.9$ and female $>0.85$ based on WHR. Patients who were still admitted and the end of the follow-up period were censored at day 30. Median time until discharge was 4 days in the group without abdominal adiposity (IQR 2-8) and 6 days in the group with abdominal adiposity (IQR 3-11). Log-rank test $p$ value $=0.129$. WHR, waist-hip ratio. 
Table 4 Univariable and multivariable Cox regression: association between patient characteristics and time until discharge

\begin{tabular}{|c|c|c|c|c|}
\hline \multirow[b]{2}{*}{ Covariate } & \multicolumn{2}{|c|}{ Univariable Cox regression } & \multicolumn{2}{|c|}{ Multivariable Cox regression } \\
\hline & HR (95\% Cl) & $P$ value & HR $(95 \% \mathrm{Cl})$ & $P$ value \\
\hline Male gender & $0.76(0.48$ to 1.22$)$ & 0.25 & $0.86(0.45$ to 1.61$)$ & 0.63 \\
\hline Age (years) & 0.99 (0.97 to 1.01$)$ & 0.48 & $0.99(0.97$ to 1.01$)$ & 0.68 \\
\hline Metabolic syndrome & 0.98 (0.60 to 1.16$)$ & 0.93 & $1.22(0.69$ to 2.15$)$ & 0.50 \\
\hline Use of antihypertensives* & 1.32 (0.80 to 2.22$)$ & 0.23 & - & - \\
\hline Hypertriglyceridemia* & $1.16(0.71$ to 1.89$)$ & 0.55 & - & - \\
\hline Low HDL-C* & 0.80 (0.40 to 1.61$)$ & 0.53 & - & - \\
\hline Hyperglycaemic* & $0.87(0.52$ to 1.45$)$ & 0.59 & - & - \\
\hline Abdominal adiposity* & 0.77 (0.47 to 1.27$)$ & 0.30 & - & - \\
\hline Waist-hip ratio & 0.98 (0.95 to 0.99$)$ & 0.04 & 0.98 (0.95 to 1.02$)$ & 0.29 \\
\hline $\mathrm{BMI}$ & 0.97 (0.93 to 1.01$)$ & 0.12 & 0.97 (0.92 to 1.01$)$ & 0.12 \\
\hline
\end{tabular}

$\mathrm{HR}<1$ related to prolonged length of stay, $\mathrm{HR}>1$ shortened length of stay. -2 Log Likelihood $499.14, \chi^{2}=7.24, p=0.203$. Statistically significant $p-$ values are bold.

*Metabolic syndrome criterion. Cut-off values: high triglycerides $(>1.7 \mathrm{mmol} / \mathrm{L})$, low HDL-C ( $<1 \mathrm{mmol} / \mathrm{L}$ in male, $<1.3 \mathrm{mmol} / \mathrm{L}$ in female), hyperglycaemia $\geq 7.8 \mathrm{mmol} / \mathrm{L}$ and/or drug treatment for elevated blood glucose, abdominal adiposity (male $\geq 102 \mathrm{~cm}$, female $\geq 88 \mathrm{~cm}$ ). $\mathrm{BMI}$, body mass index; HDL-C, high-density lipoprotein cholesterol.

were admitted to the hospital ward, whereas none of the patients without the MetS was admitted. However, the presented results do not support a relationship between MetS and an impaired clinical outcome or duration of admission by COVID-19.

This study also investigated the influence of MetS and abdominal adiposity on the hospital length of stay in patients with COVID-19. Kaplan-Meier analyses comparing patients with COVID-19 with or without MetS showed no significant differences between the groups. The Kaplan-Meier curve comparing patients with and without abdominal adiposity showed a trend towards a longer duration of hospitalisation in patients with abdominal adiposity, agreeing with the finding that abdominal adiposity promotes a more severe course of disease. In univariable analysis, abdominal adiposity was associated with a longer duration of hospitalisation in COVID-19. However, in multivariable Cox regression, there were no clinical factors associated with a prolonged duration of admission.

Several mechanisms may explain the observed relation between abdominal adiposity and respiratory failure. First, visceral fat deposits, which are increased in abdominal adiposity, act as a reservoir for viral load promoting an inflammatory response. ${ }^{2324}$ Augmented visceral fat volume is also associated with an impaired viral shedding. ${ }^{8}$ Second, an imbalance in proinflammatory and anti-inflammatory adipokines can cause increased respiratory distress. Levels of IL-6, leptin and adiponectin were determined at baseline and compared between groups, in order to test the hypothesis that proinflammatory adipokines are higher in patients with COVID-19 with MetS compared with those without MetS. The presented results, although derived from a small sample size, support the idea that the leptin-adiponectin ratio was elevated in patients with COVID-19 with MetS. No association between the leptin-adiponectin ratio on outcome in COVID-19 could be demonstrated. In addition, higher IL-6 levels were related to an unfavourable outcome. But, IL-6 was elevated in patients with COVID-19 irrespective of the presence of MetS. This might implicate that IL-6 levels in low-grade inflammation are negligible in MetS compared with the levels measured during COVID-19 infection. Earlier studies already showed that IL-6 is elevated in (severe) COVID-19 and is an independent predictor for the need of mechanical ventilation in patients with COVID-19. ${ }^{145-27}$

An alternative explanation for the relation between abdominal adiposity and respiratory failure, which was not further investigated in this study, is that increased intra-abdominal pressure due to local fat deposition may lead to mechanical obstruction and impaired ventilation of the lower lung regions. In theory, the large waist circumference may increase small airway resistance and reduce the functional residual capacity (FRC), thereby decreasing lung compliance and causing compression atelectasis. ${ }^{28}$ This has been demonstrated to be an important mechanism of action in other pulmonary conditions, such as asthma. ${ }^{29}$

In contrast to earlier large studies, hypertension or a history of cardiovascular disease, which are common comorbidities among COVID-19 and well-known risk factors for clinical deterioration, ${ }^{1} 4630$ were not associated with severe respiratory failure in our cohort. Newly discovered hypertension at the ER was not included as a criterion of MetS, due to the influence of anxiety and stress caused by COVID-19 or hospitalisation. Possibly, the use of this definition may have caused a small proportion of patients with hypertension among patients with COVID-19 in this study, explaining the discrepancy with previous reports.

The present study has a few limitations. First, the cutoff value for respiratory distress used in our study was a requirement of supplemental oxygen $\geq 3 \mathrm{~L} / \mathrm{min}$. The 


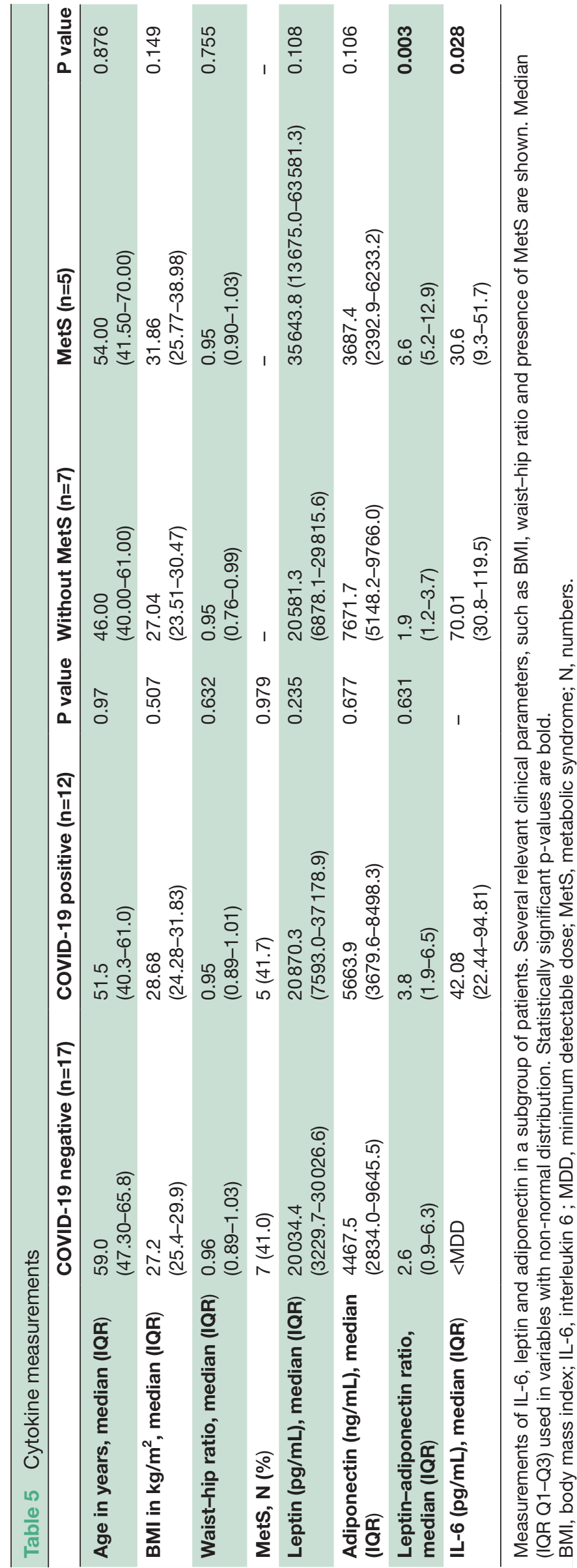



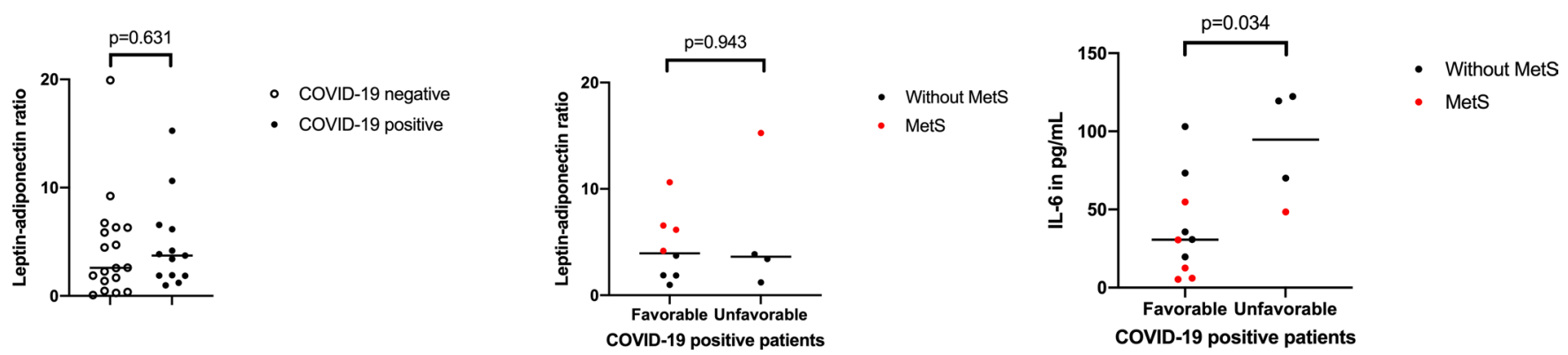

Figure 4 Distribution and comparison of leptin-adiponectin ratio between patients positive for COVID-19 and negative for COVID-19 and patients positive for COVID-19 divided in favourable and unfavourable outcome. IL-6, interleukin 6; MetS, metabolic syndrome.

rationale for this cut-off point was based on clinical experience in our centre. It was observed that the clinical condition in patients with COVID-19 often deteriorated soon after admission and that oxygen demand quickly increased in patients who required oxygen at $\geq 3 \mathrm{~L} /$ min. A cut-off value of $\geq 3 \mathrm{~L} / \mathrm{min}$ to define respiratory distress was also used in a study by Demoule et $a l .{ }^{31} \mathrm{In}$ this analysis, 257 out of 397 patients required intubation, thereby underlining the rationale for the applied cut-off value. Second, the sample size is limited, foremost in the measurement of cytokines, and documentation of clinical parameters is incomplete in some cases. However, post hoc analysis revealed a power of 0.85 for the presented model, meaning acceptable power. Finally, some bias in measurement of the waist-hip circumference could not be avoided, as they are supposed to be measured in upright position. Therefore, patients who were unable to stand at admission due to respiratory distress had to be excluded from analysis, thereby introducing selection bias. However, given the small number of patients to whom this applied (20 out of 506), the influence on the results is expected to be limited.

\section{CONCLUSION}

In the present study, MetS did not appear to be of influence on the clinical outcome in COVID-19. However, the results show a clear association between respiratory deterioration in COVID-19 and abdominal adiposity, assessed by the easily measurable waist-hip circumference. As abdominal adiposity is prevalent worldwide, ${ }^{32}$ the influence on clinical outcome in COVID-19 is equally, if not more, important than the effect of BMI. Further research with bigger sample size, extensive determination of bioinflammatory markers and lung function data (ie, FRC) could help to explain the underlying mechanisms responsible for the association between abdominal adiposity and respiratory distress in COVID-19.

\author{
Author affiliations \\ ${ }^{1}$ Pulmonology, Franciscus Gasthuis en Vlietland, Rotterdam, Zuid-Holland, \\ The Netherlands \\ ${ }^{2}$ Department of Pulmonology, Erasmus Medical Center, Rotterdam, Zuid- \\ Holland, The Netherlands
}

${ }^{3}$ Internal Medicine, Franciscus Gasthuis en Vlietland, Rotterdam, ZuidHolland, The Netherlands

${ }^{4}$ Department of Clinical Chemistry, Franciscus Gasthuis en Vlietland, Rotterdam, Zuid-Holland, The Netherlands

${ }^{5}$ Department of Statistics and Education, Franciscus Gasthuis en Vlietland, Rotterdam, Zuid-Holland, The Netherlands

${ }^{6}$ Department of Genetics, University Medical Centre Groningen, Groningen, Groningen, The Netherlands

${ }^{7}$ Department of Internal Medicine, Franciscus Gasthuis en Vlietland,

Rotterdam, Zuid-Holland, The Netherlands

Acknowledgements The authors acknowledge Ms Dr J P M Kuiper-van der Valk (Franciscus Gasthuis \& Vlietland Hospital, Department of Pulmonology) for her contribution.

Contributors CMvZ contributed to conceptualisation, study design, data collection, statistical analyses and manuscript writing. MLJ contributed to study design, data collection, statistical analyses and manuscript writing. NP contributed to laboratory analyses and manuscript review. EB contributed to review statistical analyses, statistical analyses and manuscript review. MCC contributed to study design and manuscript review. G-JB contributed to conceptualisation, study design and manuscript review.

Funding The authors have not declared a specific grant for this research from any funding agency in the public, commercial or not-for-profit sectors.

Competing interests None declared.

Patient consent for publication Not required.

Ethics approval This study was performed after approval by the Institutional Research Board of the Franciscus Gasthuis \& Vlietland, Rotterdam, the Netherlands, and was registered in the Netherlands Trial Register with reference NL8580.

Provenance and peer review Not commissioned; externally peer reviewed.

Data availability statement All data relevant to the study are included in the article or uploaded as supplementary information.

Supplemental material This content has been supplied by the author(s). It has not been vetted by BMJ Publishing Group Limited (BMJ) and may not have been peer-reviewed. Any opinions or recommendations discussed are solely those of the author(s) and are not endorsed by BMJ. BMJ disclaims all liability and responsibility arising from any reliance placed on the content. Where the content includes any translated material, BMJ does not warrant the accuracy and reliability of the translations (including but not limited to local regulations, clinical guidelines, terminology, drug names and drug dosages), and is not responsible for any error and/or omissions arising from translation and adaptation or otherwise.

Open access This is an open access article distributed in accordance with the Creative Commons Attribution 4.0 Unported (CC BY 4.0) license, which permits others to copy, redistribute, remix, transform and build upon this work for any purpose, provided the original work is properly cited, a link to the licence is given, and indication of whether changes were made. See: https://creativecommons.org/ licenses/by/4.0/.

ORCID iD

Cathelijne M van Zelst http://orcid.org/0000-0002-9221-7439 


\section{REFERENCES}

1 Richardson S, Hirsch JS, Narasimhan M, et al. Presenting characteristics, comorbidities, and outcomes among 5700 patients hospitalized with COVID-19 in the new York City area. JAMA 2020;323:2052-9.

2 Xie J, Tong Z, Guan X, et al. Clinical characteristics of patients who died of coronavirus disease 2019 in China. JAMA Netw Open 2020;3:e205619.

3 Bhatraju PK, Ghassemieh BJ, Nichols M, et al. Covid-19 in Critically III Patients in the Seattle Region - Case Series. N Engl J Med 2020;382:2012-22.

4 Grasselli G, Zangrillo A, Zanella A, et al. Baseline characteristics and outcomes of 1591 patients infected with SARS-CoV-2 admitted to ICUs of the Lombardy region, Italy. JAMA 2020;323:1574.

5 Simonnet A, Chetboun M, Poissy J, et al. High prevalence of obesity in severe acute respiratory syndrome Coronavirus-2 (SARS-CoV-2) requiring invasive mechanical ventilation. Obesity 2020;28:1195-9.

6 Rottoli M, Bernante P, Belvedere A, et al. Obesity is one of the strongest risk factor for respiratory failure and death in COVID-19. Patients: A Retrospective Multicentric Cohort Study, 2020.

7 Van Kerkhove MD, Vandemaele $\mathrm{KAH}$, Shinde V, et al. Risk factors for severe outcomes following 2009 influenza $A$ (H1N1) infection: a global pooled analysis. PLoS Med 2011;8:e1001053.

8 Maier HE, Lopez R, Sanchez N, et al. Obesity increases the duration of influenza A virus shedding in adults. $J$ Infect Dis 2018;218:1378-82.

9 Després J-P, Lemieux I. Abdominal obesity and metabolic syndrome. Nature 2006;444:881-7.

10 Yang Y, Ding L, Zou X, et al. Visceral adiposity and high intramuscular fat deposition independently predict critical illness in patients with SARS-CoV-2. Obesity 2020;28:2040-8.

11 Petersen A, Bressem K, Albrecht J, et al. The role of visceral adiposity in the severity of COVID-19: highlights from a unicenter cross-sectional pilot study in Germany. Metabolism 2020;110:154317.

12 Oda N, Imamura S, Fujita T, et al. The ratio of leptin to adiponectin can be used as an index of insulin resistance. Metabolism 2008;57:268-73.

13 López-Jaramillo P, Gómez-Arbeláez D, López-López J, et al. The role of leptin/adiponectin ratio in metabolic syndrome and diabetes. Horm Mol Biol Clin Investig 2014;18:37-45.

14 Zhang W, Zhao Y, Zhang F, et al. The use of anti-inflammatory drugs in the treatment of people with severe coronavirus disease 2019 (COVID-19): the perspectives of clinical immunologists from China. Clin Immunol 2020;214:108393.

15 Ryan PM, Caplice NM. Is adipose tissue a reservoir for viral spread, immune activation, and cytokine amplification in coronavirus disease 2019? Obesity 2020;28:1191-4.

16 Watkins J. Preventing a covid-19 pandemic. BMJ 2020;368:m810.
17 Stefan N, Birkenfeld AL, Schulze MB, et al. Obesity and impaired metabolic health in patients with COVID-19. Nat Rev Endocrinol 2020;16:341-2.

18 Grundy SM, Cleeman JI, Daniels SR, et al. Diagnosis and management of the metabolic syndrome: an American heart Association/National heart, lung, and blood Institute scientific statement. Circulation 2005;112:2735-52.

19 Waist circumference and waist-hip ratio: report of a who expert consultation, Geneva, 8-11 who library Cataloguing-in-Publication data, 2008. Available: https://apps.who.int/iris/bitstream/handle/ 10665/44583/9789241501491 eng.pdf?sequence=1December

20 Volksgezondheidenzorg.info. Prevalence of overweight and obesity among the adult population in the Netherlands Bilthoven, the Netherlands: RIVM (Netherlands Institute of Public Health \& Environment). Available: https://www.volksgezondheidenzorg.info/ onderwerp/overgewicht/regionaal-internationaal/regionaal

21 Paolini J-BM, Mancini J, Genestal M, et al. Predictive value of abdominal obesity vs. body mass index for determining risk of intensive care unit mortality. Crit Care Med 2010;38:1308-14.

22 Pischon T, Boeing H, Hoffmann K, et al. General and abdominal adiposity and risk of death in Europe. $N$ Engl J Med 2008;359:2105-20.

23 Peters MC, Fahy JV. Metabolic consequences of obesity as an "outside in" mechanism of disease severity in asthma. Eur Respir $J$ 2016:48:291-3.

24 de Lucena TMC, da Silva Santos AF, de Lima BR, et al. Mechanism of inflammatory response in associated comorbidities in COVID-19. Diabetes Metab Syndr 2020;14:597-600.

25 Herold T, Jurinovic V, Arnreich C, et al. Elevated levels of IL-6 and CRP predict the need for mechanical ventilation in COVID-19. $J$ Allergy Clin Immunol 2020;146:128-36.

26 Ruan Q, Yang K, Wang W, et al. Clinical predictors of mortality due to COVID-19 based on an analysis of data of 150 patients from Wuhan, China. Intensive Care Med 2020;46:846-8

27 Wu C, Chen X, Cai Y, et al. Risk factors associated with acute respiratory distress syndrome and death in patients with coronavirus disease 2019 pneumonia in Wuhan, China. JAMA Intern Med 2020;180:934-43.

28 Steele RM, Finucane FM, Griffin SJ, et al. Obesity is associated with altered lung function independently of physical activity and fitness. Obesity 2009;17:578-84.

29 van Huisstede A, Rudolphus A, Castro Cabezas M, et al. Effect of bariatric surgery on asthma control, lung function and bronchial and systemic inflammation in morbidly obese subjects with asthma. Thorax 2015;70:659-67.

30 Guan W-J, Ni Z-Y, Hu Y, et al. Clinical characteristics of coronavirus disease 2019 in China. N Engl J Med 2020;382:1708-20.

31 Demoule A, Vieillard Baron A, Darmon M, et al. High flow nasal Canula in critically ill severe COVID-19 patients. Am J Respir Crit Care Med 2020.

32 Eckel RH, Grundy SM, Zimmet PZ. The metabolic syndrome. Lancet 2005;365:1415-28. 